\title{
Risperidone Versus Methylphenidate in Treatment of Preschool Children With Attention-Deficit Hyperactivity Disorder
}

\author{
Fariba Arabgol $^{1, *} ;$ Leily Panaghi $^{2}$; Vahid Nikzad ${ }^{3}$ \\ ${ }_{1}^{1}$ Behavioral Science Research Center, Shahid Beheshti University of Medical Sciences, Tehran, IR Iran \\ ${ }^{2}$ Family Research Institute, Shahid Beheshti University, Tehran, IR Iran \\ 3 Bronx-Lebanon Hospital Center, Albert Einstein College of Medicine, New York, USA \\ *Corresponding author: Fariba Arabgol, Behavioral Science Research Center, Shahid Beheshti University of Medical Sciences, Tehran, IR Iran. E-mail: farabgol@sbmu.ac.ir
}

Received:September 11, 2014; Revised: December 1, 2014; Accepted: January 10, 2015

\begin{abstract}
Background: Attention Deficit Hyperactivity Disorder (ADHD) is a common psychiatric diagnosis among preschool children. Objectives: The aim of this study was to examine the Risperidone treatment compared to Methylphenidate(MPH) in preschool children with ADHD.

Patients and Methods: Thirty three outpatient preschool children, aged 3-6 years, diagnosed with ADHD (The diagnosis of ADHD was established by two child and adolescent psychiatrists according to the DSM-IV-TR criteria), participated in a 6-week, double-blind clinical trial with risperidone $(0.5-1.5 \mathrm{mg} / \mathrm{d})$ and methylphenidate $(5-20 \mathrm{mg} / \mathrm{d})$, in two divided doses. Treatment outcomes were assessed using the Parent ADHD Rating Scale and Conners Rating Scale. Patients were assessed by a child psychiatrist at baseline, 2, 4 and 6 weeks after the medication started. Side effects were also rated by side effects questionnaire.

Results: There were no significant differences between the two protocols on the Parent ADHD Rating Scale scores $(P>0.05)$ and Parent Conners Rating Scale scores $(\mathrm{P}>0.05)$. Both groups showed a significant improvement in ADHD symptoms over the 6 weeks of treatment for parent ADHD Rating Scale $(\mathrm{P}<0.001)$ and Parent Conners Rating Scale $(\mathrm{P}<0.001)$. The most common adverse effects seen with risperidone were daytime drowsiness and anorexia (20\%), and with methylphenidate it was anorexia (55\%).

Conclusions: Results of this study show that risperidone may be effective and well tolerated for ADHD in preschool children, but more researches are needed to clarify the potential benefits and adverse effects in long term use and comorbid conditions.
\end{abstract}

Keywords: Attention Deficit Hyperactivity Disorder; Methylphenidate; Risperidone; Preschool Children

\section{Background}

Attention deficit hyperactivity disorder, ADHD (1) is one of the most common childhood onset psychiatric disorders, affecting $5-12 \%$ of children worldwide (2). It can cause significant impairment in all areas of functioning in patients of all ages (3). It is important to note that impairment due to ADHD will continue into adulthood and will require treatment (4). Preschool ADHD prevalence rates vary from a low of $2 \%$ in the primary care office to a high of 59\% in a child psychiatry clinic (5). The level of impairment of preschoolers with ADHD is similar to that of affected school age children (6). But more specifically, poor academic readiness for school entry, poor fine motor skills, and social impairment are more common in preschoolers with ADHD relative to healthy controls (7). Other problems include preschool expulsion, difficulty in maintaining babysitters or childcare and as a result, missed family work days and personal time (8).

Systematic research and practice guidelines about psychopharmacotherapy in very young children are limited (9), Methylphenidate is the first-line psychopharmacological treatment for preschool ADHD in the Preschool Psychopharmacology Working Group (PPWG) algorithm
(9) but the Preschool Attention-Deficit/Hyperactivity Disorder Treatment Study (PATS) showed that effect size in preschoolers is smaller than in older children $(9,10)$. This group also had higher rates of emotional adverse effects including lability, crabbiness, irritability, and proneness to crying compared to older children (4-11). Also preschool children with ADHD may experience slightly more and different types of stimulant-induced side effects compared with older children (5).

Two other classes of medication, A-agonists (such as clonidine) and atomoxetine are commonly used for treatment of ADHD (9), but there is little evidence about safety and efficacy of atomoxetine in preschoolers ADHD. In a double-blind, placebo-controlled, randomized clinical trial about atomoxetine in ADHD children aged 5-6 years, authors found that despite benefits, the children in the atomoxetine group remained significantly impaired at the end of the trial (12). A-agonists are more commonly used to treat ADHD in preschoolers $(13,14)$ and this group of drugs can be associated with adverse effects including sedation, irritability, bradycardia, and hypotension $(15,16)$, and also regular moni-

Copyright (C) 2015, Growth \& Development Research Center. This is an open-access article distributed under the terms of the Creative Commons Attribution-NonCommercial 4.0 International License (http://creativecommons.org/licenses/by-nc/4.0/) which permits copy and redistribute the material just in noncommercial usages, provided the original work is properly cited. 
toring of blood pressure and heart rate is required (9). In overdose they may induce sedation, hypotension, or death (17). Thus, inability to administer and store the medication safely may be a contraindication to using A-agonists (9).

On the other hand, several investigations have found high co-morbidity rates of disruptive disorders with ADHD (18-22). For example, oppositional defiant disorder (ODD) is co-morbid with ADHD in 60\% of referred preschoolers $(6,23)$. Studies have shown that co-morbidity of ODD with ADHD is associated with other poor outcomes such as a higher rate of injuries when compared to those with ADHD alone (20). The use of atypical antipsychotics have been risen significantly for treating disruptive behavior disorders in children (22) and positive effects of atypical antipsychotics for disruptive behavior has increased the use of them in the attention-deficit hyperactivity disorder population (24). Aman et al. (25) studied the safety and efficacy of risperidone in the presence and absence of psychostimulant medicine in children. They found that in a subgroup of Disruptive Behavior Disorders (DBD) and sub average Intelligence Quotient (IQ) patients with co-morbid ADHD risperidone-treated patients had significant reductions in both disruptive behavior and hyperactivity symptoms, compared to placebo, regardless of concomitant stimulant use, but addition of risperidone to stimulant resulted in significantly better control of hyperactivity than stimulant treatment alone, and this combination didn't increase adverse effects (25).

In a review article about second-generation antipsychotic medications in children and adolescents, the authors found that the majority (43\%) of the studies were on risperidone, and one of the disorders in children in which atypical antipsychotics are potentially useful is severe attention deficit hyperactivity disorder (26), and also risperidone is safe and effective in treating DBDs in children (27). For these reasons, we need alternative pharmacological treatment of ADHD in preschoolers and this study was conducted to compare risperidone versus methylphenidate in preschool children with ADHD.

\section{Objectives}

The aim of this study was to examine the Risperidone treatment compared to Methylphenidate (MPH) in preschool children with ADHD.

\section{Patients and Methods}

This study was a randomized clinical trial conducted at the clinic of Child Psychiatry of Imam Hossein hospital affiliated to Shahid Beheshti Medical University in Tehran, Iran. The parents were informed about procedures and purposes of the study and their consent obtained. The study was approved by the hospital's Institutional Review Board and Iranian Randomized Clinical Trial.

\subsection{Subjects}

Thirty three outpatient preschoolers with ADHD participated in the study. The diagnosis of ADHD was established by two child and adolescent psychiatrists according to the DSM-IV-TR criteria (1). Exclusion criteria were the presence of any physical disease, mental retardation and any psychiatric co-morbid disorders except conduct disorder and oppositional defiant disorder. Patients were randomized to receive risperidone or methylphenidate. The randomization and allocation process was done by the resident of pediatric psychiatry of Imam Hossein Hospital. All subjects were randomly assigned to receive treatment using either methylphenidate (group 1) or risperidone (group 2) for a 6-week double-blind clinical trial. All subjects were new patients with no drug history since 2 weeks ago and they did not receive other drugs or psychological intervention during the intervention phase.

\subsection{Design and Procedures}

Risperidone was started at a dose of $0.25 \mathrm{mg}$ per day in one dose and gradually (every week) increased based on the therapeutic response and the patient's tolerance. The optimal dose of risperidone was $2 \mathrm{mg}$ /day in two divided doses. The mean daily dosage of risperidone at the end of 6 weeks was $0.89 \pm 0.48 \mathrm{mg} /$ day. The dose was chosen according to the effective dose of risperidone and its side effects from previous studies of risperidone in preschoolers (28-30). Methylphenidate was started at a dose of 2.5 mg per day and gradually (every week) increased based on the therapeutic response and the patient's tolerance. The optimal dose of methylphenidate was $20 \mathrm{mg} /$ day in two divided doses. The dose was chosen according to the prior studies $(5,9)$. The mean dose of methylphenidate was $12.83 \pm 0.56 \mathrm{mg} /$ day.

Throughout the study one child and adolescent psychiatrist adjusted the dose of medications based on the clinical response and adverse effects observed. Questionnaires were rated by the other clinician simultaneously. The person who administrated the medications, the rater, and the patients were blind to the assignments. The principal measures of the outcome were the Parent ADHD Rating Scale (31) and Conners Revised Parent Rating Scale (32). Patients were assessed by a child and adolescent psychiatrist at baseline $\left(\mathrm{T}_{0}\right) ; \mathrm{T}_{1}, 2$ weeks; $\mathrm{T}_{2}, 4$ weeks and $T_{3}, 6$ weeks after the medication started. Side effects of the drugs were assessed using the risperidone and methylphenidate Side Effects Form.

\subsection{Statistical Analysis}

The two independent sample t-tests were used for comparison of the age, parent ADHD rating scale and Conners Revised Parent Rating Scale at baseline in two groups. Chi square test was used for comparison of genders between the two groups and dropouts groups. Mann-Whitney test was used for comparison of age in dropout groups. 
To compare frequency of side effects between the two protocols, two sided Fisher's exact test was applied. Twoway repeated measures analysis of variance (time $\times$ treatment interaction) was done for Parent ADHD Rating Scale scores and Conners Parent Rating Scale. The two groups as a between-subjects factor (group) and the four measurements during treatment as the within-subjects factor (time) were considered.

\section{Results}

Thirty eight outpatients preschoolers with ADHD (27 boys and 11 girls) aged 3 to 6 (mean \pm SD, $4.42 \pm 0.82$ ) participated in the study. Thirty three of 38 (86.84\%) participants, 15 participants in Methylphenidate group and 18 participants in Risperidone group completed the treatment protocol. Three patients from methylphenidate group and two patients from risperidone group discontinued the treatment. Nineteen of $33(57.57 \%)$ participants were combined subtype of ADHD, 11 (33.33\%) hyperactive/impulsive and $3(9.09 \%)$ inattentive (Table 1$)$. No significance differences were identified between group 1 or 2 with regard to age $(\mathrm{t}=2.04, \mathrm{df}=31, \mathrm{P}=0.06)$, ADHD subtypes $(\mathrm{df}=2, \mathrm{P}=$ $0.9, \chi^{2}=0.2$ ) and gender $\left(\mathrm{df}=1, \mathrm{P}=0.24, \chi^{2}=1.38\right)$ (Table 1 ).

No significant differences were observed between the two protocols on the Parent ADHD Rating Scale $(t=0.72$, $\mathrm{df}=31, \mathrm{P}=0.47)$ and Conners Parent Rating Scale $(\mathrm{t}=0.12$, $\mathrm{df}=31, \mathrm{P}=0.06$ ) scores in Time 0 , before intervention (Tables 2 and 3). Repeated measures of ANOVA, one for Parent ADHD Rating Scale, two for its subscales and one for Conners Parent Rating Scale were done.

\subsection{Parent ADHD Rating Scale}

For parent ADHD Rating Scale, The within subject effect (time) was significant $(\mathrm{F}=45.25, \mathrm{df}=1.72,48.37, \mathrm{P}<0.001$ ), but neither interaction (time and group of the treatment) ( $\mathrm{F}=0.14, \mathrm{df}=1,28, \mathrm{P}>0.05)$ nor between subject effect (group of the treatment) were significant $(F=0.01$, $\mathrm{df}=1.72,48.37, \mathrm{P}>0.05$ (Table 2, Figure 1). It means during the therapeutic intervention there is a significant response in both groups from time 0 and time 3 , but there is no significant difference between the two groups.

\begin{tabular}{lcc}
\hline Table 1. Demographic Data ${ }^{\mathrm{a}}$ & $\begin{array}{c}\text { Risperidone } \\
\text { Group }\end{array}$ & $\begin{array}{c}\text { Methylphenidate } \\
\text { group }\end{array}$ \\
\hline Variables & 7 & 3 \\
\hline Fender & 11 & 12 \\
\hline Male & & \\
ADHD Subtypes & 10 & 9 \\
Combined & 6 & 5 \\
Hyperactive/Impulsive & 2 & 1 \\
\hline Inattentive & $4.17 \pm 0.79$ & $4.73 \pm 0.77$ \\
Age, $\mathbf{y}$ & $0.89 \pm 0.48$ & $12.83 \pm 0.56$ \\
\hline Dosage &
\end{tabular}

${ }^{\mathrm{a}}$ Data are presented as No. or mean \pm SD.
Table 2. Mean (SD) Scores for Parent ADHD Rating Scales and Subscales $\mathrm{a}, \mathrm{b}, \mathrm{c}$

\begin{tabular}{|c|c|c|}
\hline \multirow[t]{2}{*}{ Variables } & \multicolumn{2}{|c|}{ Groups of treatment } \\
\hline & Risperidone & Methylphenidate \\
\hline \multicolumn{3}{|c|}{ Parent ADHD Rating Scale } \\
\hline $\mathrm{T}_{0}$ & $29.16 \pm 6.59$ & $27.60 \pm 5.72$ \\
\hline $\mathrm{T}_{1}$ & $21.66 \pm 7.91$ & $21.13 \pm 5.81$ \\
\hline $\mathrm{T}_{2}$ & $19.50 \pm 8.94$ & $18.53 \pm 6.71$ \\
\hline $\mathrm{T}_{3}$ & $16.64 \pm 9.53$ & $15.53 \pm 6.30$ \\
\hline \multicolumn{3}{|c|}{ Inattentive Subscale } \\
\hline $\mathrm{T}_{0}$ & $12.33 \pm 4.20$ & $11.46 \pm 3.04$ \\
\hline $\mathrm{T}_{1}$ & $9.11 \pm 4.22$ & $9.13 \pm 2.85$ \\
\hline $\mathrm{T}_{2}$ & $8.44 \pm 3.58$ & $8.13 \pm 3.56$ \\
\hline $\mathrm{T}_{3}$ & $7.58 \pm 4.50$ & $6.84 \pm 3.64$ \\
\hline \multicolumn{3}{|c|}{$\begin{array}{l}\text { Hyperactive/Impulsive } \\
\text { Subscale }\end{array}$} \\
\hline $\mathrm{T}_{0}$ & $16.55 \pm 4.24$ & $16.13 \pm 4.17$ \\
\hline $\mathrm{T}_{1}$ & $12.50 \pm 5.03$ & $12.00 \pm 4.01$ \\
\hline $\mathrm{T}_{2}$ & $11.27 \pm 6.42$ & $10.40 \pm 4.18$ \\
\hline $\mathrm{T}_{3}$ & $9.00 \pm 5.97$ & $8.69 \pm 4.21$ \\
\hline \multicolumn{3}{|c|}{$\begin{array}{l}\text { a All values are based on mean } \pm \text { SD. } \\
\mathrm{b}_{\text {There is no significant difference between groups at } \mathrm{T}_{0}, \mathrm{~T}_{1}, \mathrm{~T}_{2}, \mathrm{~T}_{3} \text { in }} \\
\text { parent ADHD rating scales and subscales. } \\
\mathrm{C}^{\mathrm{C}} \mathrm{T}_{0}=\text { Baseline, } \mathrm{T}_{1}=2 \text { Weeks After the Treatment, } \mathrm{T}_{2}=4 \text { Weeks After the } \\
\text { Treatment, } \mathrm{T}_{3}=6 \text { Weeks After the Treatment. }\end{array}$} \\
\hline
\end{tabular}

Table 3. Mean (SD) Scores for Conners Parent Rating ScaleRevised ${ }^{\mathrm{a}, \mathrm{b}, \mathrm{c}}$

\begin{tabular}{lcc}
\hline Variables & \multicolumn{2}{c}{ Groups of treatment } \\
\cline { 2 - 3 } & \multicolumn{1}{c}{ Risperidone } & Methylphenidate \\
\hline $\begin{array}{l}\text { Conners Parent Rating } \\
\text { Scale }\end{array}$ & $54.61 \pm 17.37$ & $55.26 \pm 11.58$ \\
$\mathrm{~T}_{0}$ & $40.72 \pm 17.94$ & $43.60 \pm 15.71$ \\
$\mathrm{~T}_{1}$ & $36.22 \pm 17.55$ & $37.46 \pm 16.28$ \\
$\mathrm{~T}_{2}$ & $30.76 \pm 19.20$ & $31.69 \pm 18.43$ \\
$\mathrm{~T}_{3}$ & \\
\hline $\mathrm{a}$ All Values are Based on Mean $\pm \mathrm{SD}$. \\
$\mathrm{b}$ There is no significant difference between groups at $\mathrm{T}_{0}, \mathrm{~T}_{1}, \mathrm{~T}_{2}, \mathrm{~T}_{3}$ in \\
Conners Parent Rating Scale. \\
${ }_{\mathrm{C}} \mathrm{T}_{0}=$ baseline, $\mathrm{T}_{1}=2$ weeks after the treatment, $\mathrm{T}_{2}=4$ weeks after the \\
treatment, $\mathrm{T}_{3}=6$ weeks after the treatment.
\end{tabular}

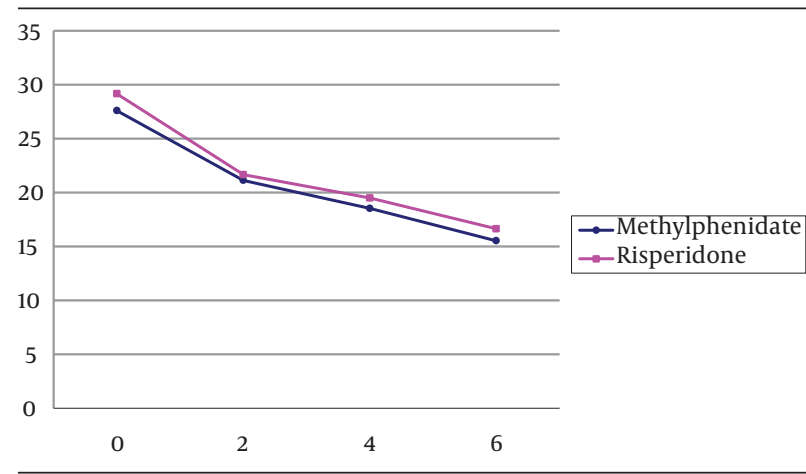

Figure 1. Changes in Parent ADHD Rating Scale 
The study power was less than 0.8 that demonstrates low sample size to find probably differences between groups. Sixty percent of decrease in parent ADHD rating scale score had occurred in the second week of treatment for risperidone group. This rate was $53.2 \%$ for methylphenidate group.

\subsection{Inattentive Subscale}

For Inattentive Subscale only the within subject effect was significant $(\mathrm{F}=35.35, \mathrm{df}=1.83,51.39, \mathrm{P}<0.001)$. But neither interaction (time and group of the treatment) ( $\mathrm{F}=0.28, \mathrm{df}=1.28, \mathrm{P}>0.05)$ nor between subject effect (group of the treatment) was significant $(\mathrm{F}=0.38, \mathrm{df}=$ 1.83, 51.39, P > 0.05) (Table 2, Figure 2).

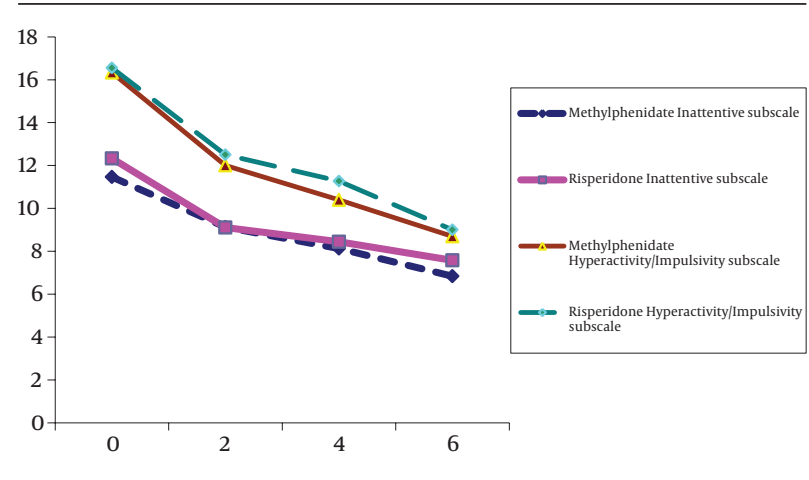

Figure 2. Changes in Parent ADHD Rating subscales

\subsection{Hyperactive/Impulsive Subscale}

For Hyperactive/Impulsive Subscale only the within subject effect was meaningful $(\mathrm{F}=36.99, \mathrm{df}=1.67,46.81$, $\mathrm{P}<0.001$ ) (Figure 3). But neither interaction (time and group of the treatment $)(\mathrm{F}=0.19, \mathrm{df}=1.67,46.81, \mathrm{P}>0.05)$ nor between subject effect (group of the treatment) was significant $(\mathrm{F}=0.01, \mathrm{df}=1.28, \mathrm{P}>0.05)$ (Table 2, Figure 2).

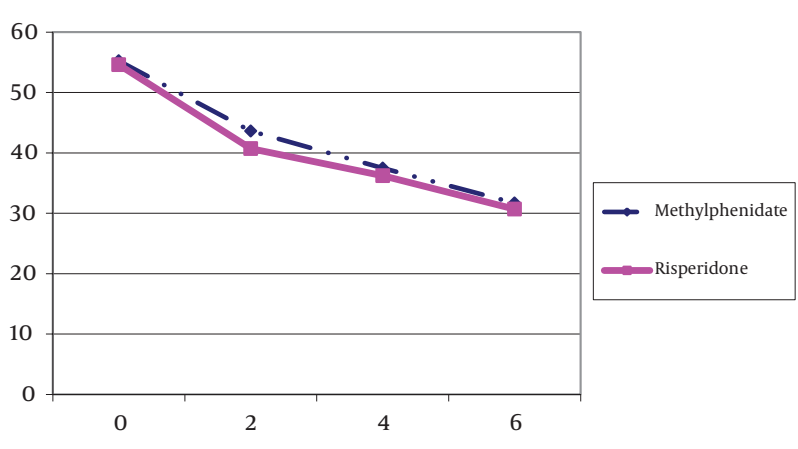

Figure 3. Changes in Conners Parent Rating scale

\subsection{Conners Parent Rating Scale-Revised}

For Parent Conners Scale only the within subject effect was meaningful $(\mathrm{F}=44.71, \mathrm{df}=1.61,45.26, \mathrm{P}<0.001)$ (Fig- ure 3). But neither interaction (time and group of the treatment $)(\mathrm{F}=0.05, \mathrm{df}=1.61,45.26, \mathrm{P}>0.05)$ nor between subject effect (group of the treatment) was significant ( $\mathrm{F}$ $=0.01, \mathrm{df}=1,28, \mathrm{P}>0.05$ ) (Table 3, Figure 3). Fifty eight percent of decrease in Conners parent rating scale score had occurred in the second week of treatment for risperidone group. This rate was $49.4 \%$ for methylphenidate group.

\subsection{Dropouts and Adverse Effects}

No significant difference was observed in two the groups regarding to the rate of dropout. Risperidone treatment was relatively well tolerated. Eighteen of 20 (90\%) participants completed the course of treatment within six weeks. Two patients discontinued treatment due to side effects. The first one was a 4-year-old boy who discontinued drug after one week of treatment because of increased restlessness and hyperactivity and the second one was a 3.5 years old boy who discontinued drug after 10 days due to sedation, polyuria and constipation. The most common side effects were daytime drowsiness and anorexia in $20 \%$ of risperidone group. In methylphenidate group fifteen of 18 (83.33\%) participants completed the treatment and $3(16.66 \%)$ patients discontinued it after one or two weeks. One 4.5-year-old girl discontinued who drug due to severe anorexia in the second week of treatment. One 5-year-old boy discontinued it because of crying and sadness in the second week of treatment and another 5-year-old boy discontinued the drug due to increased symptoms and aggression. The most common side effects were anorexia (55.55\%), nervousness (33.33\%) and disturbed sleeping (27.77\%) in methylphenidate group.

\section{Discussion}

This double-blind, randomized and controlled study suggests risperidone is effective in alleviating total symptoms of ADHD and related co-morbid symptoms in preschooler and that there is no statistically significant difference between the two groups in alleviating ADHD symptoms. Our results are partially in agreement with previous studies that have shown a positive effect of risperidone in treatment of ADHD symptoms (33). In Correia's study, risperidone induced more reductions in ADHD total score than methylphenidate, but there are some differences between two studies. The subjects in Correia's study were ADHD children and adolescents with moderate mental retardation, not preschool normal IQ patients, as our subjects were (33). Our study is also partially in agreement with Gunther's study, in that they found clinical symptoms of ADHD and DBD significantly improved after a 4-week treatment period with risperidone, although they studied children aged 8-15 years and here is a difference of subjects' age (34). Also our study is in agreement with Ercan's study about risperidone effects for the treatment of normally developing preschool children with diagnosis of Conduct Disorder (CD) co-morbid 
with ADHD. Result of this open label trial shows that risperidone significantly improved symptoms and subscales of symptoms such as inattention subscale score (28). Although we used a different questionnaire, in our study there was significant improvement based on Conners scale that measures CD, ODD, ADHD as well as other symptoms. Ercan et al. measured symptoms with Turgay DSM-IV Based Disruptive Behavior Disorders Child and Adolescent Screening and Rating Scale that assesses inattention, hyperactivity-impulsivity, opposition-defiance and conduct disorder. Indeed both questionnaires assess same symptoms (28).

Our finding shows risperidone treatment was relatively well tolerated and the most common side effect was daytime drowsiness similar to that in previous studies $(25,26,34,35)$. We did not study metabolic adverse effects and prolactine level to which previous studies were concerned about $(28,36)$. Also some previous researches reported higher body weight be associated with antipsychotic treatment in younger aged children (37). It seems that in future studies we need to investigate long term side effects of risperidone in preschool children. In methylphenidate group fifteen of 18 (83.33\%) participants accomplished the treatment and $3(16.66 \%)$ patients discontinued the treatment after one or two weeks, that is in relative agreement with Wigal study in which eleven percent of subjects discontinued MPH because of adverse events (11). Although our study shows that risperidone alleviates ADHD symptoms and is well tolerated in preschoolers, regarding metabolic and extrapyramidal side effects, these should be evaluated precisely and its use limited to patients which do not respond to stimulants or show intolerable side effects.

Limitations and suggestion: Our study assessed a relatively small sample of patients in a short-term intervention. ADHD is a generally chronic neuropsychiatric condition that may last many years, we do not know whether therapeutic effects of risperidone last for a long term or not. Also further investigations are needed to assess long-term safety when risperidone is prescribed for very young children, regarding the possibility of adverse effects on the developing brain. Also trials are needed to evaluate eventual metabolic and cardiovascular adverse effects of risperidone in very young children. We recommend further future studies to assess the therapeutic efficacy of risperidone in preschooler ADHD with different co-morbidities.

\section{Acknowledgements}

We would like to thank all the participants for their cooperation. We also are grateful for Behavioral Sciences Research Center affiliated with Shahid Beheshti Medical University for funding the study.

\section{References}

1. APA. Diagnostic and statistical manual of mental disorders. 4th ed.
Text revised (DSM-VI-TR). editor. Washington:American Psychiatric Association; 2000.

2. Arnold LE, Spetie L. Attention-Deficit/Hyperactivity Disorder. 4th ed. Martin AF editor. : Lippincott Williams \& Wilkins; 2007.

3. Waslick B, Greenhill L. Attention-deficit/Hyperactivity Disorder. 3rd ed. Wiener JM editor. : American Psychiatry Publishing Inc;2004.

4. Pliszka S, Aacap Work Group on Quality Issues . Practice parameter for the assessment and treatment of children and adolescents with attention-deficit/hyperactivity disorder. $J$ Am Acad Child Adolesc Psychiatry. 2007;46(7):894-921.

5. Connor DF. Preschool attention deficit hyperactivity disorder: a review of prevalence, diagnosis, neurobiology, and stimulant treatment. J Dev Behav Pediatr. 2002;23(1 Suppl):S1-9.

6. Wilens TE, Biederman J, Brown S, Tanguay S, Monuteaux MC, Blake C, Spencer TJ, et al. Psychiatric comorbidity and functioning in clinically referred preschool children and school-age youths with ADHD. J Am Acad Child Adolesc Psychiatry. 2002;41(3):262-8.

7. DuPaul GJ, McGoey KE, Eckert TL, VanBrakle J. Preschool children with attention-deficit/hyperactivity disorder: impairments in behavioral, social, and school functioning. J Am Acad Child Adolesc Psychiatry. 2001;40(5):508-15.

8. Tandon M, Si X, Luby J. Preschool onset attention-deficit/hyperactivity disorder: course and predictors of stability over 24 months. J Child Adolesc Psychopharmacol. 2011;21(4):321-30.

9. Gleason MM, Egger HL, Emslie GJ, Greenhill LL, Kowatch RA, Lieberman AF, Luby JL, Owens J, Scahill LD, Scheeringa MS, Stafford $\mathrm{B}$, Wise B, Zeanah $\mathrm{CH}$, et al. Psychopharmacological treatment for very young children: contexts and guidelines. J Am Acad Child Adolesc Psychiatry. 2007;46(12):1532-72.

10. Greenhill L, Kollins S, Abikoff H, McCracken J, Riddle M, Swanson J, McGough J, Wigal S, Wigal T, Vitiello B, Skrobala A, Posner K, Ghuman J, Cunningham C, Davies M, Chuang S, Cooper T, et al. Efficacy and safety of immediate-release methylphenidate treatment for preschoolers with ADHD. J Am Acad Child Adolesc Psychiatry. 2006;45(11):1284-93.

11. Wigal T, Greenhill L, Chuang S, McGough J, Vitiello B, Skrobala A, Swanson J, Wigal S, Abikoff H, Kollins S, McCracken J, Riddle M, Posner K, Ghuman J, Davies M, Thorp B, Stehli A, et al. Safety and tolerability of methylphenidate in preschool children with ADHD. J Am Acad Child Adolesc Psychiatry. 2006;45(11):1294-303.

12. Kratochvil CJ, Vaughan BS, Stoner JA, Daughton JM, Lubberstedt BD, Murray DW, Chrisman AK, Faircloth MA, Itchon-Ramos NB, Kollins SH, Maayan LA, Greenhill LL, Kotler LA, Fried J, March JS, et al. A double-blind, placebo-controlled study of atomoxetine in young children with ADHD. Pediatrics. 2011;127(4):e862-8.

13. Rappley MD, Mullan PB, Alvarez FJ, Eneli IU, Wang J, Gardiner JC. Diagnosis of attention-deficit/hyperactivity disorder and use of psychotropic medication in very young children. Arch Pediatr Adolesc Med.1999;153(10):1039-45.

14. Zito JM, Safer DJ, dosReis S, Gardner JF, Boles M, Lynch F. Trends in the prescribing of psychotropic medications to preschoolers. JAMA. 2000;283(8):1025-30.

15. Connor DF, Fletcher KE, Swanson JM. A meta-analysis of clonidine for symptoms of attention-deficit hyperactivity disorder. $J$ Am Acad Child Adolesc Psychiatry. 1999;38(12):1551-9.

16. Scahill L, Chappell PB, Kim YS, Schultz RT, Katsovich L, Shepherd E, Arnsten AF, Cohen DJ, Leckman JF, et al. A placebo-controlled study of guanfacine in the treatment of children with tic disorders and attention deficit hyperactivity disorder. Am J Psychiatry. 2001;158(7):1067-74.

17. Klein-Schwartz W. Trends and toxic effects from pediatric clonidine exposures. Arch Pediatr Adolesc Med. 2002;156(4):392-6.

18. Gadow KD, Nolan EE. Differences between preschool children with ODD, ADHD, and ODD+ADHD symptoms.J Child Psychol Psychiatry. 2002;43(2):191-201.

19. Keenan K. Emotion Dysregulation as a Risk Factor for Child Psychopathology. Clin Psychol-Sci Pr. 2006;7(4):418-34.

20. Schwebel DC, Speltz ML, Jones K, Bardina P. Unintentional injury in preschool boys with and without early onset of disruptive behavior. J Pediatr Psychol. 2002;27(8):727-37.

21. Wakschlag LS, Briggs-Gowan MJ, Carter AS, Hill C, Danis B, Keenan K, McCarthy KJ, Leventhal BL, et al. A developmental framework for distinguishing disruptive behavior from norma- 
tive misbehavior in preschool children. J Child Psychol Psychiatry. 2007;48(10):976-87.

22. Loy JH, Merry SN, Hetrick SE, Stasiak K. Atypical antipsychotics for disruptive behaviour disorders in children and youths. Cochrane Database Syst Rev. 2012;9.

23. Wilens TE, Biederman J, Brown S, Monuteaux M, Prince J, Spencer TJ. Patterns of psychopathology and dysfunction in clinically referred preschoolers. J Dev Behav Pediatr. 2002;23(1 Suppl):S31-6.

24. Weiss M, Panagiotopoulos C, Giles L, Gibbins C, Kuzeljevic B, Davidson J, Harrison R, et al. A naturalistic study of predictors and risks of atypical antipsychotic use in an attention-deficit/hyperactivity disorder clinic. J Child Adolesc Psychopharmacol. 2009;19(5):575-82.

25. Aman MG, Binder C, Turgay A. Risperidone effects in the presence/ absence of psychostimulant medicine in children with ADHD, other disruptive behavior disorders, and subaverage IQ. J Child Adolesc Psychopharmacol.2004;14(2):243-54.

26. Cheng-Shannon J, McGough JJ, Pataki C, McCracken JT. Secondgeneration antipsychotic medications in children and adolescents. J Child Adolesc Psychopharmacol. 2004;14(3):372-94.

27. Reyes M, Croonenberghs J, Augustyns I, Eerdekens M. Long-term use of risperidone in children with disruptive behavior disorders and subaverage intelligence: efficacy, safety, and tolerability. $J$ Child Adolesc Psychopharmacol. 2006;16(3):260-72.

28. Ercan ES, Basay BK, Basay O, Durak S, Ozbaran B. Risperidone in the treatment of conduct disorder in preschool children with out intellectual disability. Child Adolesc Psychiatry Ment Health. 2011;5(1):10.

29. Biederman J, Mick E, Hammerness P, Harpold T, Aleardi M, Dougherty M, Wozniak J, et al. Open-label, 8-week trial of olanzapine and risperidone for the treatment of bipolar disorder in preschool-age children. Biol Psychiatry. 2005;58(7):589-94.
30. Findling RL, McNamara NK, Branicky LA, Schluchter MD, Lemon E, Blumer JL. A double-blind pilot study of risperidone in the treatment of conduct disorder. J Am Acad Child Adolesc Psychiatry. 2000;39(4):509-16.

31. Dupaul GJ. Parent and Teacher Ratings of ADHD Symptoms: Psychometric Properties in a Community-Based Sample. J Clin Child Psychol.1991;20(3):245-53.

32. Conners CK. Conners Rating Scales.New York: Multi-Health Systems Inc.; 1997.

33. Correia Filho AG, Bodanese R, Silva TL, Alvares JP, Aman M, Rohde LA. Comparison of risperidone and methylphenidate for reducing ADHD symptoms in children and adolescents with moderate mental retardation. J Am Acad Child Adolesc Psychiatry. 2005;44(8):748-55.

34. Gunther T, Herpertz-Dahlmann B, Jolles J, Konrad K. The influence of risperidone on attentional functions in children and adolescents with attention-deficit/hyperactivity disorder and co-morbid disruptive behavior disorder. J Child Adolesc Psychopharmacol. 2006;16(6):725-35.

35. Eapen V, Gururaj AK. Risperidone treatment in 12 children with developmental disorders and attention-deficit/hyperactivity disorder. Prim Care Companion J Clin Psychiatry. 2005;7(5):221-4.

36. Stadler C, Bolten M, Schmeck K. Pharmacotherapeutic intervention in impulsive preschool children: The need for a comprehensive therapeutic approach. Child Adolesc Psychiatry Ment Health. 2011;5(1):11

37. Gebhardt S, Haberhausen M, Heinzel-Gutenbrunner M, Gebhardt N, Remschmidt H, Krieg JC, Hebebrand J, Theisen FM, et al. Antipsychotic-induced body weight gain: predictors and a systematic categorization of the long-term weight course. J Psychiatr Res. 2009;43(6):620-6. 